\title{
Relationship of serum bilirubin concentrations to kidney function and albuminuria in the United States adult population. Findings from the National Health and Nutrition Examination Survey 2001-2006
}

\author{
Giovanni Targher ${ }^{1, *}$, Cortney Bosworth², \\ Jessica Kendrick ${ }^{2}$, Gerard Smits ${ }^{2}$, Giuseppe \\ Lippi $^{3}$ and Michel Chonchol ${ }^{2}$ \\ ${ }^{1}$ Section of Endocrinology, Department of \\ Biomedical and Surgical Sciences, University of \\ Verona, Verona, Italy \\ ${ }^{2}$ Division of Renal Diseases and Hypertension, \\ University of Colorado Denver Health Sciences \\ Center, Denver, CO, USA \\ ${ }^{3}$ Section of Clinical Chemistry, Department of \\ Biomedical and Morphological Sciences, University \\ Hospital of Verona, Verona, Italy
}

\begin{abstract}
Background: The association of serum bilirubin concentrations with kidney function and albuminuria has not been established in the US general population. Methods: We performed a cross-sectional analysis of data from the National Health and Nutrition Examination Survey (NHANES) 2001-2006 and examined the associations of serum total bilirubin concentrations with estimated glomerular filtration rate (eGFR) and albuminuria in a nationally representative sample of 13,184 adults aged 20 years or older. eGFR was estimated using the abbreviated Modification of Diet in Renal Disease equation. Urinary albumin excretion was measured using the albumin/creatinine ratio.

Results: An eGFR $<60 \mathrm{~mL} / \mathrm{min} / 1.73 \mathrm{~m}^{2}$ and urinary albumin/creatinine ratio $\geq 30 \mathrm{mg} / \mathrm{g}$ were present in $8.1 \%(n=1072)$ and $10.6 \%(n=1402)$ of participants, respectively. A total of 427 (3.2\%) individuals had a serum total bilirubin concentration $>22.23 \mu \mathrm{mol} / \mathrm{L}$. After adjustment for demographics, comorbidities, alcohol consumption, viral hepatitis status and other laboratory measures, serum bilirubin was negatively associated with eGFR, and positively with albuminuria both in the whole cohort and in the subgroup of non-diabetic individuals. In contrast, serum bilirubin was not significantly associated with eGFR or albuminuria in persons with diabetes $(n=1253)$.

Conclusions: Increasing serum concentrations of total bilirubin are independently associated with decreas-

\footnotetext{
* Corresponding author: Dr. Giovanni Targher, Section of Endocrinology, Department of Biomedical and Surgical Sciences, University of Verona, Ospedale Civile Maggiore, Piazzale Stefani, 1, 37126 Verona, Italy Phone: +39-045-8123748, Fax: +39-045-8027314, E-mail: giovanni.targher@univr.it Received March 17, 2009; accepted June 5, 2009; previously published online July 27, 2009
}

ing eGFR and increasing albuminuria in the US adult population.

Clin Chem Lab Med 2009;47:1055-62.

Keywords: albuminuria; bilirubin; epidemiology; estimated glomerular filtration rate; liver dysfunction.

\section{Introduction}

The prevalence of chronic kidney disease (CKD) is increasing worldwide $(1,2)$. Reduced estimated glomerular filtration rate (eGFR) and abnormal albuminuria have been associated with increased risk of end-stage renal disease, cardiovascular disease and other comorbidities (3-6). Because kidney disease often progresses to end-stage renal disease, the identification of precursors and risk factors for kidney disease progression are essential, with the belief that interventions will prevent or delay progression to kidney failure.

Increased concentrations of serum bilirubin have long been used as a marker of liver dysfunction. In addition, serum bilirubin has been shown to be inversely related to cardiovascular disease, suggesting a potentially athero-protective effect of bilirubin (7-10). However, the relationship of serum bilirubin with the future risk of cardiovascular events appears to be more complex. A U-shaped relationship was observed between serum bilirubin and risk of major ischemic heart disease events in a prospective study of 7685 middle-aged British men who were followed for 11.5 years (11). It is also known that serum bilirubin concentrations are significantly higher in men than in women, and tend to decline with advancing age in both sexes (12).

Information on the association of serum bilirubin concentrations with kidney function and albuminuria is limited and controversial. Fukui et al. found that serum total bilirubin was positively associated with eGFR, and negatively with albuminuria in a hospitalbased sample of 633 Japanese type 2 diabetic patients (13). These results suggest a potential renoprotective effect of bilirubin. In contrast, we found that higher serum total bilirubin was significantly associated with lower eGFR in both non-diabetic and diabetic individuals in 2678 unselected outpatients 35 years or age or older (14).

To date, the association of serum bilirubin concentrations with kidney function and albuminuria have 
not been established in the US general population. We, therefore, examined the association of serum bilirubin concentrations with kidney function measures in the National Health and Nutrition Examination Survey (NHANES) 2001-2006, a nationally representative cross-sectional examination of the US adult civilian population.

\section{Materials and methods}

\section{Study population}

The NHANES is a program designed to assess the health and nutritional status of the US civilian non-institutionalized population. The study was approved by the National Center for Health Statistics (NCHS) Institutional Review Board and all subjects gave informed consent (15). The NHANES examinations were conducted from 2001 to 2006 in three phases (2001-2002, 2003-2004 and 2005-2006), and data from these phases were combined for the purpose of this analysis, following NCHS analytical guidelines (16).

In all phases of NHANES, a stratified, multistage sampling design was used, with over-sampling of non-Hispanic blacks, Mexican-Americans and persons over the age of 60 years. Individuals participated in an interview conducted at home, as well as an extensive physical examination performed at a mobile examination center, which included blood and urine collection (17). This analysis was initially restricted to 13,213 adults 20 years of age or older. However, responders who had a missing serum bilirubin and incomplete information on the main covariates of interest were excluded from analysis $(n=29)$. Thus, 13,184 adults constituted the sample used in this analysis.

\section{Study variables}

After collection of fasting, venous blood samples were immediately centrifuged. Specimens were then frozen and shipped weekly to a central laboratory where they were initially stored at $-20^{\circ} \mathrm{C}$, and then at $-70^{\circ} \mathrm{C}(16,17)$. Serum total bilirubin was determined by automated biochemical profiling (Beckman Synchron LX20, Beckman Coulter, Fullerton, CA, USA); fractionation of bilirubin was not performed. The LX20 uses a timed-endpoint Diazo method to measure the concentration of serum total bilirubin. The analytical range for this assay is $1.71-513 \mu \mathrm{mol} / \mathrm{L}(0.1-30 \mathrm{mg} / \mathrm{dL})$, and the reference range is $3.42-22.23 \mu \mathrm{mol} / \mathrm{L}(0.2-1.3 \mathrm{mg} / \mathrm{dL})$. Both intra- and inter-assay coefficients of variation were $<3 \%$ (16).

eGFR was derived from the re-expressed MDRD equation $=175.0 \times(\text { serum creatinine value })^{-1.154} \times$ age $^{-0.203} \times 0.742$ (if female) $\times 1.21$ (if black) (18). Values that exceeded 200 $\mathrm{mL} / \mathrm{min} / 1.73 \mathrm{~m}^{2}$ were truncated. Serum creatinine was measured using the Jaffé method (kinetic alkaline picrate) with a Beckman Synchron LX20 $(16,19)$. As reported in the NHANES laboratory procedure manual, hemolysis, lipemia and bilirubin $<34.2 \mu \mathrm{mol} / \mathrm{L}(<2 \mathrm{mg} / \mathrm{dL})$ have no significant interference with the measurement of serum creatinine. At bilirubin levels $>51.3 \mu \mathrm{mol} / \mathrm{L}(>3 \mathrm{mg} / \mathrm{dL}$ ), the creatinine value is decreased by $6.84 \mu \mathrm{mol} / \mathrm{L}(0.4 \mathrm{mg} / \mathrm{dL})$. Other potential interfering substances (e.g., cefoxitin, cefaclor, gluthathione, acetoacetic acid, L-DOPA methyl ester) are reported in detail in the laboratory procedures manual $(16,19)$. Both intra- and inter-assay coefficients of variation were $<3.5 \%$. As recommended by NHANES analytic guidelines (19), NHANES serum creatinine values in 2005-2006 were adjusted to ensure comparability with standard creatinine using the following formula: standard creatinine $(\mathrm{mg} / \mathrm{dL})=-0.016+$ $0.978 \times$ (NHANES 2005-2006 uncalibrated serum creatinine $[\mathrm{mg} / \mathrm{dL}])$. However, a recent calibration study in which the original serum creatinine measurements (Jaffé method) in NHANES 2001-2002, and 2003-2004 were compared with standard creatinine measured using an assay traceable to known gold-standard methods implemented at the Cleveland Clinic Research Laboratory demonstrated that no correction was needed for serum creatinine concentrations measured in NHANES 2001-2004 (20). In particular, the Cleveland Clinic Research laboratory analyzed the serum creatinine specimens using a Roche coupled enzymatic assay (creatininase, creatinase, sarcosine oxidase, kits \#1775677 and \#1775766) performed on a Roche P Module instrument (Roche Diagnostics, Basel, Switzerland). The Roche method calibrators were traceable to an isotope dilution mass spectrometric method for serum creatinine using standard references methods (NIST-SRM 967). They were also confirmed by analysis of CAP LN-24 linearity set based on National Institute of Standards and Technology (NIST) assigned values (20).

Urinary albumin excretion was measured using a fluorescent immunoassay (Sequoia-Turner model 450 digital fluorometer, Block Scientific, Holbrook, NY, USA) on the basis of the spot urine albumin/creatinine ratio. Both intra- and inter-assay coefficients of variation were $<9 \%$ (16). Abnormal albuminuria was defined as albumin/creatinine ratio $\geq 30 \mathrm{mg} / \mathrm{g}$ (18).

Serum concentrations of total cholesterol, high-density lipoprotein (HDL) cholesterol, triglycerides, alanine aminotransferase (ALT), aspartate aminotransferase (AST) and $\gamma$ glutamyltransferase (GGT) were measured enzymatically with a Hitachi-704 Analyzer (Roche Diagnostics, Indianapolis, IN, USA) (16). Low-density lipoprotein (LDL) cholesterol was calculated using the Friedewald's equation, except when triglycerides exceeded $4.55 \mathrm{mmol} / \mathrm{L}$. Exposure to hepatitis C virus (HCV) was determined by presence of antibody to HCV, and hepatitis $B$ infection was identified by the presence of hepatitis B core antibody (16). Serum glucose was measured using a modified hexokinase enzymatic method, and a radioimmunoassay method was used to measure serum insulin (16). Homeostasis model assessment of insulin resistance (HOMA-IR) was calculated using fasting glucose and insulin measurements as follows [ fasting insulin $(\mu \mathrm{U} / \mathrm{mL}) \times$ fasting glucose $(\mathrm{mmol} / \mathrm{L}) / / 22.5]$ (21). HOMA-IR score was available in non-diabetic individuals.

Self-reported race was defined as non-Hispanic white, non-Hispanic black, Mexican-American, or other. A diagnosis of hypertension was assigned if the subject reported a physician diagnosis of hypertension, if the subject reported taking prescription medications for hypertension, or if the systolic blood pressure was $\geq 140 \mathrm{~mm} \mathrm{Hg}$ or the diastolic blood pressure was $\geq 90 \mathrm{~mm} \mathrm{Hg}$. Participants were defined as having diabetes if they were taking hypoglycemic drugs, had a fasting plasma glucose concentration $\geq 7 \mathrm{mmol} / \mathrm{L}$ or if a physician told them that they had diabetes. Smoking status was classified as never smoked, ex-smoker or current smoker. Alcohol consumption was recorded as number of drinks per day. Body mass index (BMI) was calculated as weight in kilograms divided by the square of height in meters. Waist circumference was measured with a steel measuring tape to the nearest $0.1 \mathrm{~cm}$ at the high point of the iliac crest at minimal respiration (16).

\section{Statistical analysis}

NHANES uses a complex, multistage, probability-sampling design to select participants that are representative of the 
civilian, non-institutionalized US population. All analyses accounted for the complex sampling method. Analyses were performed with SAS version 9.1 (SAS Institute Inc., Cary, NY, USA) callable SUDAAN version 9.01 (Research Triangle Institute, Research Triangle Park, NC, USA).

Results are expressed as mean \pm SD or percentages. For statistical purposes (Tables 1 and 2), participants were stratified into categories of eGFR (i.e., $<60,60-89$ and $\geq 90$ $\mathrm{mL} / \mathrm{min} / 1.73 \mathrm{~m}^{2}$ ) and albuminuria (classified as normal and abnormal, i.e., albumin/creatinine ratio $\geq 30 \mathrm{mg} / \mathrm{g}$ ). In these analyses, we combined subjects with eGFR $15-30 \mathrm{~mL} / \mathrm{min} /$ $1.73 \mathrm{~m}^{2}$ and those with eGFR $30-59 \mathrm{~mL} / \mathrm{min} / 1.73 \mathrm{~m}^{2}$ into a single eGFR category due to the low number of individuals $(\mathrm{n}=66)$ with eGFR values of $15-30 \mathrm{~mL} / \mathrm{min} / 1.73 \mathrm{~m}^{2}$.

Statistical analyses included one-way analysis of variance (for continuous variables) and the $\chi^{2}$-test with Yates's correction for continuity (for categorical measures). Skewed variables (triglycerides and HOMA-IR score) were logarithmically transformed to improve normality prior to analysis.

The association between serum bilirubin and eGFR (included as continuous variable) was investigated using linear (unadjusted and fully adjusted) regression models in the whole group and in subgroups of participants stratified by diabetes status (Table 3 ). We excluded subjects who had a serum creatinine concentration $>176.8 \mu \mathrm{mol} / \mathrm{L}$ ( $>2.0$ $\mathrm{mg} / \mathrm{dL})$ or serum bilirubin concentrations $>22.23 \mu \mathrm{mol} / \mathrm{L}$ $(>1.3 \mathrm{mg} / \mathrm{dL})(\mathrm{n}=513)$. The covariates included in fullyadjusted regression models were age, gender, race/ethnicity, smoking status, hypertension, diabetes, BMI, waist circumference, total cholesterol, LDL-cholesterol, HDL-cholesterol, triglycerides, alcohol consumption, viral hepatitis markers, glycosylated hemoglobin, HOMA-IR score and albuminuria.

The association between serum bilirubin and albuminuria (included as categorical variable) was investigated using logistic regression models in the whole group, excluding subjects who had a serum creatinine $>176.8 \mu \mathrm{mol} / \mathrm{L}(>2.0$ $\mathrm{mg} / \mathrm{dL})$ or serum bilirubin $>22.23 \mu \mathrm{mol} / \mathrm{L}(>1.3 \mathrm{mg} / \mathrm{dL})$ $(n=513)$, and in subgroups of participants stratified by diabetes status (Table 4). The covariates included in fullyadjusted regression models were age, gender, race/ethnicity, smoking status, hypertension, diabetes, BMI, waist circumference, total cholesterol, LDL-cholesterol, HDL-cholesterol, triglycerides, alcohol consumption, viral hepatitis markers, glycosylated hemoglobin, insulin resistance and eGFR.

In all regression models, observations were weighted to reflect the general US population as of the early 2000 s, using

Table 1 Age-gender standardized baseline demographics, comorbidities and laboratory results according to eGFR in the entire cohort $(n=13,184)$.

\begin{tabular}{|c|c|c|c|c|}
\hline eGFR & \multirow{2}{*}{$\begin{array}{l}\geq 90 \mathrm{~mL} / \mathrm{min} / 1.73 \mathrm{~m}^{2} \\
\mathrm{n}=6987\end{array}$} & \multirow{2}{*}{$\begin{array}{l}60-89 \mathrm{~mL} / \mathrm{min} / 1.73 \mathrm{~m}^{2} \\
\mathrm{n}=5125\end{array}$} & \multirow{2}{*}{$\begin{array}{l}<60 \mathrm{~mL} / \mathrm{min} / 1.73 \mathrm{~m}^{2} \\
\mathrm{n}=1072\end{array}$} & \multirow{2}{*}{$\begin{array}{l}p \text {-Value } \\
\text { for trend }\end{array}$} \\
\hline Characteristics & & & & \\
\hline Age, years & $38(14)$ & $52(16)$ & $71(10)$ & $<0.0001$ \\
\hline Gender, \% & & & & 0.01 \\
\hline Males & 47.4 & 48.9 & 36.4 & \\
\hline Females & 52.6 & 51.1 & 63.6 & \\
\hline Ethnicity, \% & & & & $<0.0001$ \\
\hline Non-Hispanic white & 39.3 & 65.1 & 71.2 & \\
\hline Non-Hispanic black & 24.7 & 15.0 & 14.0 & \\
\hline Mexican-American & 31.5 & 16.5 & 11.3 & \\
\hline Other & 4.5 & 3.4 & 3.5 & \\
\hline Smoking status, $\%$ & & & & $<0.0001$ \\
\hline Never & 53.4 & 51.1 & 50.9 & \\
\hline Prior & 18.8 & 27.9 & 38.5 & \\
\hline Current & 27.8 & 21.0 & 10.6 & \\
\hline Hypertension, \% & 19.5 & 34.5 & 68.1 & $<0.0001$ \\
\hline Diabetes, $\%$ & 6.5 & 8.4 & 23.7 & $<0.0001$ \\
\hline Alcohol drinks per day, \% & & & & $<0.0001$ \\
\hline 0 & 36.1 & 36 & 57.6 & \\
\hline $1-3$ & 44.8 & 51.9 & 39.7 & \\
\hline$>3$ & 19.1 & 12.1 & 2.7 & \\
\hline \multicolumn{5}{|l|}{ Measurements } \\
\hline BMI, $\mathrm{kg} / \mathrm{m}^{2}$ & $28(7)$ & $29(6)$ & $29(5)$ & 0.001 \\
\hline Waist circumference, inches & $38(7)$ & $39(6)$ & $40(5)$ & $<0.0001$ \\
\hline Glucose, $\mathrm{mmol} / \mathrm{L}$ & $5.28(1.8)$ & $5.44(1.8)$ & $6.11(2.0)$ & $<0.0001$ \\
\hline Total cholesterol, $\mathrm{mmol} / \mathrm{L}$ & $5.12(1.1)$ & $5.26(1.1)$ & $5.25(1.1)$ & $<0.0001$ \\
\hline LDL cholesterol, mmol/L & $2.96(0.9)$ & $3.01(0.9)$ & $2.98(0.8)$ & 0.0005 \\
\hline HDL cholesterol, $\mathrm{mmol} / \mathrm{L}$ & $1.38(0.4)$ & $1.38(0.4)$ & $1.37(0.4)$ & 0.27 \\
\hline Triglycerides, mmol/L & $1.66(1.8)$ & $1.70(1.4)$ & $1.93(1.0)$ & 0.0003 \\
\hline AST, U/L & $25(23)$ & $25(13)$ & $26(11)$ & 0.60 \\
\hline $\mathrm{ALT}, \mathrm{U} / \mathrm{L}$ & $26(26)$ & $25(15)$ & $22(11)$ & 0.001 \\
\hline GGT, U/L & $29(55)$ & $30(34)$ & $30(47)$ & 0.17 \\
\hline Total bilirubin, $\mu \mathrm{mol} / \mathrm{L}$ & $12.3(5.4)$ & $12.8(4.6)$ & $13.0(6.8)$ & $<0.0001$ \\
\hline Glycosylated hemoglobin, \% & $5.5(1.0)$ & $5.6(0.9)$ & $5.9(0.9)$ & $<0.0001$ \\
\hline HOMA-IR score & $3.3(3.4)$ & $3.5(3.9)$ & $4.8(5.2)$ & $<0.0001$ \\
\hline Albuminuria, $\%$ & 8.1 & 10.0 & 30.1 & $<0.0001$ \\
\hline Hepatitis B antibody, \% & 20.5 & 16.1 & 11.5 & $<0.0001$ \\
\hline Hepatitis C antibody, \% & 1.0 & 0.9 & 0.5 & 0.20 \\
\hline
\end{tabular}

Results are expressed as means $( \pm S D)$ or percentages. ALT, alanine aminotransferase; AST, aspartate aminotransferase; BMI, body mass index; GGT, $\gamma$-glutamyltransferase; HDL, high-density lipoprotein; HOMA-IR, homeostasis model assessment of insulin resistance; LDL, low-density lipoprotein; eGFR, estimated glomerular filtration rate. 
Table 2 Age-gender standardized baseline demographics, comorbidities and laboratory results according to urinary albumin excretion in the entire cohort $(n=13,184)$.

\begin{tabular}{|c|c|c|c|}
\hline Urine albumin/creatinine ratio, mg/g & & $\geq 30$ & $\mathrm{p}$-Value \\
\hline Characteristics & $\mathrm{n}=11,652$ & $n=1532$ & for difference \\
\hline Age, years & $44(17)$ & $54(17)$ & $<0.0001$ \\
\hline Gender, \% & & & 0.002 \\
\hline Males & 47.8 & 43.3 & \\
\hline Females & 52.2 & 56.7 & \\
\hline Ethnicity, \% & & & $<0.0001$ \\
\hline Non-Hispanic white & 51.3 & 42.8 & \\
\hline Non-Hispanic black & 20.0 & 25.3 & \\
\hline Mexican-American & 24.7 & 27.3 & \\
\hline Other & 4.0 & 4.6 & \\
\hline Smoking status, \% & & & 0.14 \\
\hline Never & 52.8 & 49.3 & \\
\hline Prior & 22.9 & 26.4 & \\
\hline Current & 24.3 & 24.3 & \\
\hline Hypertension, \% & 25.2 & 50.6 & $<0.0001$ \\
\hline Diabetes, \% & 6.0 & 26.6 & $<0.0001$ \\
\hline Alcohol drinks per day, \% & & & $<0.0001$ \\
\hline 0 & 35.8 & 47.0 & \\
\hline $1-3$ & 48.2 & 39.9 & \\
\hline$>3$ & 16.0 & 13.1 & \\
\hline eGFR, \%, mL/min/1.73 $\mathrm{m}^{2}$ & & & $<0.0001$ \\
\hline$\geq 90$ & 59.1 & 46.2 & \\
\hline $60-89$ & 36.7 & 36.0 & \\
\hline$<60$ & 4.2 & 17.8 & \\
\hline \multicolumn{4}{|l|}{ Measurements } \\
\hline $\mathrm{BMI}, \mathrm{kg} / \mathrm{m}^{2}$ & $28(6)$ & $30(8)$ & $<0.0001$ \\
\hline Waist circumference, inches & $38(6)$ & $40(6)$ & $<0.0001$ \\
\hline Glucose, $\mathrm{mmol} / \mathrm{L}$ & $5.22(1.5)$ & $6.61(3.2)$ & $<0.0001$ \\
\hline Total cholesterol, mmol/L & $5.18(1.1)$ & $5.26(1.2)$ & 0.03 \\
\hline LDL cholesterol, mmol/L & $2.97(0.9)$ & $3.02(0.9)$ & 0.02 \\
\hline HDL cholesterol, mmol/L & $1.38(0.4)$ & $1.36(0.4)$ & 0.13 \\
\hline Triglycerides, mmol/L & $1.65(1.5)$ & $2.04(2.3)$ & $<0.0001$ \\
\hline $\mathrm{AST}, \mathrm{U} / \mathrm{L}$ & $25(19)$ & $26(14)$ & 0.24 \\
\hline $\mathrm{ALT}, \mathrm{U} / \mathrm{L}$ & $26(22)$ & $25(15)$ & 0.05 \\
\hline GGT, U/L & $29(39)$ & $40(81)$ & $<0.0001$ \\
\hline Total bilirubin, $\mu \mathrm{mol} / \mathrm{L}$ & $12.4(4.9)$ & $12.3(6.3)$ & 0.40 \\
\hline Glycosylated hemoglobin, \% & $5.5(0.8)$ & $6.3(1.7)$ & $<0.0001$ \\
\hline HOMA-IR score & $3.3(3.4)$ & $5.1(5.8)$ & $<0.0001$ \\
\hline Hepatitis B antibody, \% & 18.5 & 16.4 & 0.06 \\
\hline Hepatitis C antibody, \% & 0.9 & 1.5 & 0.02 \\
\hline
\end{tabular}

Results are expressed as means $( \pm S D)$ or percentages. eGFR, estimated glomerular filtration rate; ALT, alanine aminotransferase; AST, aspartate aminotransferase; BMI, body mass index; GGT, $\gamma$-glutamyltransferase; HDL, high-density lipoprotein; HOMA-IR, homeostasis model assessment of insulin resistance; LDL, low-density lipoprotein.

weights calculated for that purpose by the National Health Statistics (17). p-Values $<0.05$ were considered significant.

\section{Results}

Among the 13,184 adult participants, the mean serum total bilirubin was $12.48( \pm 5.3) \mu \mathrm{mol} / \mathrm{L}$, the median was 12.0 (inter-quartile range $10.26-13.68) \mu \mathrm{mol} / \mathrm{L}$. A total of 427 (3.2\%) persons had a serum bilirubin concentration $>22.23 \mu \mathrm{mol} / \mathrm{L}(>1.3 \mathrm{mg} / \mathrm{dL})$. An eGFR $<60 \mathrm{~mL} / \mathrm{min} / 1.73 \mathrm{~m}^{2}$ or albumin/creatinine ratio $\geq 30 \mathrm{mg} / \mathrm{g}$ were present in $8.1 \%(\mathrm{n}=1072)$ and $10.6 \%$ $(n=1402)$ of the whole sample, respectively.

The clinical and biochemical characteristics of participants stratified by eGFR categories are summarized in Table 1. Compared with those with normal or near-normal eGFR, persons with lower eGFR were older, more likely to be female, more likely to be nonHispanic white, and had greater prevalence of hypertension, diabetes and abnormal albuminuria, and higher values of BMI, waist circumference, serum glucose, glycosylated hemoglobin, insulin resistance, total cholesterol, LDL-cholesterol, triglycerides, and total bilirubin concentrations. Moreover, participants with lower eGFR also had lower serum ALT activity and were less likely to report alcohol consumption and a lower prevalence of hepatitis B seropositivity.

When participants were stratified by categories of albuminuria (Table 2), the results remained essentially unchanged with a single important exception, i.e., no significant differences were found in serum bilirubin concentrations between participants with normal and those with abnormal albuminuria. Moreover, 
Table 3 Association of serum bilirubin concentrations with eGFR in NHANES participants.

\begin{tabular}{|c|c|c|c|c|}
\hline & $\beta(95 \% \mathrm{Cl})$ & Standardized $\beta$ & t-Value & p-Value \\
\hline \multicolumn{5}{|l|}{ Whole cohort $(n=13,184)$} \\
\hline Unadjusted & $-8.35(-9.79$ to -6.91$)$ & -0.10 & -11.4 & $<0.0001$ \\
\hline Multivariate adjusted & $-3.40(-4.71$ to -2.09$)$ & -0.04 & -5.12 & $<0.0001$ \\
\hline \multicolumn{5}{|c|}{$\begin{array}{l}\text { Excluding participants with a } \\
\text { serum creatinine }>2.0 \mathrm{mg} / \mathrm{dL} \text { or } \\
\text { bilirubin }>1.3 \mathrm{mg} / \mathrm{dL}(\mathrm{n}=12,671)\end{array}$} \\
\hline Unadjusted & $-16.22(-18.30$ to -14.14$)$ & -0.14 & -15.3 & $<0.0001$ \\
\hline Multivariate adjusted & $-8.41(-10.35$ to -6.47$)$ & -0.07 & -8.51 & $<0.0001$ \\
\hline \multicolumn{5}{|l|}{ Non-diabetics $(n=11,931)$} \\
\hline Unadjusted & $-9.35(-10.82$ to -7.88$)$ & -0.11 & -12.50 & $<0.0001$ \\
\hline Multivariate adjusted ${ }^{\mathrm{b}}$ & $-4.12(-5.45$ to -2.79$)$ & -0.05 & -6.09 & $<0.0001$ \\
\hline \multicolumn{5}{|l|}{ Diabetics $(n=1253)$} \\
\hline Unadjusted & $1.61(-4.88$ to 8.10$)$ & 0.01 & 0.5 & 0.63 \\
\hline Multivariate adjusted $^{\mathrm{C}}$ & $2.06(-5.07$ to 9.19$)$ & 0.01 & 0.6 & 0.57 \\
\hline
\end{tabular}

${ }^{a}$ Adjusted for age, gender, race/ethnicity, smoking, hypertension, diabetes, body mass index, waist circumference, total cholesterol, low-density lipoprotein cholesterol, high-density lipoprotein cholesterol, triglycerides, alcohol consumption, viral hepatitis markers, glycosylated hemoglobin, albuminuria, and homeostasis model assessment of insulin resistance. ${ }^{\mathrm{b}} \mathrm{Adjusted}$ for age, gender, race/ethnicity, smoking, hypertension, diabetes, body mass index, waist circumference, total cholesterol, lowdensity lipoprotein cholesterol, high-density lipoprotein cholesterol, triglycerides, alcohol consumption, viral hepatitis markers, homeostasis model assessment of insulin resistance, and albuminuria. ' $A d j u s t e d$ for age, gender, race/ethnicity, smoking, hypertension, diabetes, body mass index, waist circumference, glucose, total cholesterol, low-density lipoprotein cholesterol, high-density lipoprotein cholesterol, triglycerides, alcohol consumption, viral hepatitis markers, glycosylated hemoglobin and albuminuria. $\mathrm{Cl}$, confidence interval; eGFR, estimated glomerular filtration rate; NHANES, National Health and Nutrition Examination Survey.

Table 4 Association of serum bilirubin concentrations with urinary albumin excretion $\geq 30 \mathrm{mg} / \mathrm{g}$ in NHANES participants.

\begin{tabular}{|c|c|c|}
\hline & Odds ratio $(95 \% \mathrm{Cl})$ & $\mathrm{p}$-Value \\
\hline \multicolumn{3}{|l|}{ Whole cohort $(n=13,184)$} \\
\hline Unadjusted & $0.92(0.75-1.12)$ & 0.40 \\
\hline Multivariate adjusted ${ }^{a}$ & $1.26(1.03-1.54)$ & 0.02 \\
\hline \multicolumn{3}{|c|}{$\begin{array}{l}\text { Excluding participants with a serum creatinine } \\
>2.0 \mathrm{mg} / \mathrm{dL} \text { or bilirubin }>1.3 \mathrm{mg} / \mathrm{dL}(\mathrm{n}=12,671)\end{array}$} \\
\hline Unadjusted & $0.88(0.67-1.15)$ & 0.35 \\
\hline Multivariate adjusted ${ }^{a}$ & $1.32(0.95-1.85)$ & 0.09 \\
\hline \multicolumn{3}{|l|}{ Non-diabetics $(n=11,931)$} \\
\hline Unadjusted & $1.05(0.85-1.29)$ & 0.68 \\
\hline Multivariate adjusted & $1.24(1.01-1.54)$ & 0.04 \\
\hline \multicolumn{3}{|l|}{ Diabetics $(n=1253)$} \\
\hline Unadjusted & $1.04(0.63-1.73)$ & 0.88 \\
\hline Multivariate adjusted ${ }^{c}$ & $1.37(0.76-2.48)$ & 0.30 \\
\hline
\end{tabular}

${ }^{a}$ Adjusted for age, gender, race/ethnicity, smoking, hypertension, diabetes, body mass index, waist circumference, total cholesterol, low-density lipoprotein cholesterol, high-density lipoprotein cholesterol, triglycerides, alcohol consumption, viral hepatitis markers, glycosylated hemoglobin, homeostasis model assessment of insulin resistance, and estimated glomerular filtration rate. ${ }^{\text {b} A d j u s t e d ~ f o r ~ a g e, ~ g e n d e r, ~ r a c e / e t h n i c i t y, ~ s m o k i n g, ~ h y p e r t e n s i o n, ~ d i a b e t e s, ~ b o d y ~ m a s s ~ i n d e x, ~ w a i s t ~ c i r c u m f e r-~}$ ence, total cholesterol, low-density lipoprotein cholesterol, high-density lipoprotein cholesterol, triglycerides, alcohol consumption, viral hepatitis markers, homeostasis model assessment of insulin resistance, and estimated glomerular filtration rate. ${ }^{\mathrm{C} A d j u s t e d}$ for age, gender, race/ethnicity, smoking, hypertension, diabetes, body mass index, waist circumference, glucose, total cholesterol, low-density lipoprotein cholesterol, high-density lipoprotein-cholesterol, triglycerides, alcohol consumption, viral hepatitis markers, glycosylated hemoglobin, and estimated glomerular filtration rate. Cl, confidence interval; NHANES, National Health and Nutrition Examination Survey.

persons with abnormal albuminuria also had higher serum GGT activity and higher prevalence of hepatitis C seropositivity.

When the analyses described above were limited to persons diagnosed with diabetes $(n=1253)$, serum total bilirubin concentrations did not change across categories of eGFR $(11.8 \pm 4.4$ vs. $11.3 \pm 3.6$ vs. $12.0 \pm 3.6 \mu \mathrm{mol} / \mathrm{L}$ from the $1 \mathrm{st}-3 \mathrm{rd}$ eGFR category, respectively; $p=0.95$ for trend) and albuminuria (11.6 \pm 3.9 vs. $11.8 \pm 3.8 \mu \mathrm{mol} / \mathrm{L}$ normal vs. abnormal albuminuria; $p=0.87$ ).
When we performed a subgroup analysis of participants stratified by age groups (Figure 1), serum bilirubin concentrations significantly increased across eGFR categories, i.e., with higher serum bilirubin in participants with lower eGFR ( $p<0.05$ or less for each age group). In contrast, serum bilirubin concentrations did not significantly change across albuminuria categories stratified by age groups (data not shown).

Table 3 shows the association between serum total bilirubin and eGFR (included as continuous variable) in univariate and multivariate linear regression mod- 


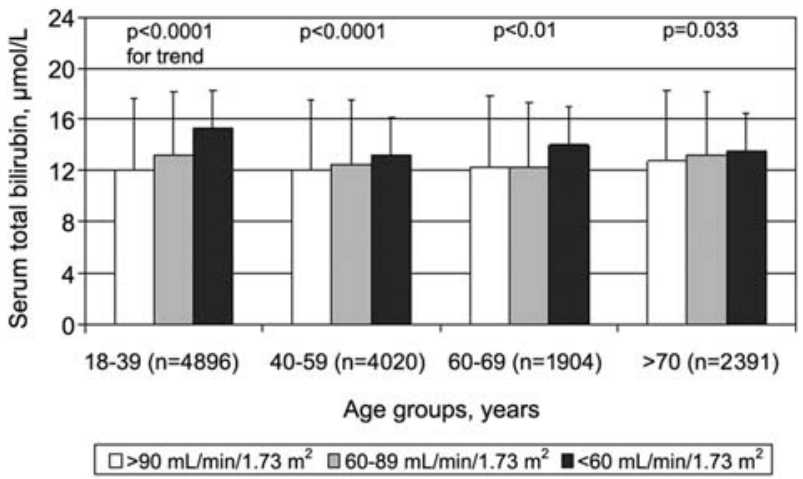

Figure 1 Mean $( \pm S D$ ) serum bilirubin concentrations by age and estimated glomerular filtration rate (eGFR) categories in the NHANES participants $(n=13,184)$.

Differences for trends in serum bilirubin for each age group were tested by one-way analysis of variance.

els. In univariate analysis, total bilirubin was inversely associated with eGFR in the whole sample, in the nondiabetic subgroup, and after excluding subjects with a serum creatinine $>176.8 \mu \mathrm{mol} / \mathrm{L}$ or a total bilirubin concentration $>22.23 \mu \mathrm{mol} / \mathrm{L}$. No significant association was found between serum bilirubin and eGFR in the diabetic subgroup. These results remained essentially unchanged after adjustment for demographics, comorbidities, alcohol consumption, viral hepatitis status, albuminuria and other laboratory measures.

Table 4 shows the association between serum total bilirubin and albuminuria - included as categorical measure - in unadjusted and fully adjusted logistic regression models. Serum bilirubin did not correlate with abnormal albuminuria in unadjusted logistic regression models, neither in the whole cohort nor in other subgroups of participants. Conversely, the association between serum bilirubin and abnormal albuminuria became significant after adjustment for demographics, comorbidities, alcohol consumption, viral hepatitis status, eGFR and other laboratory measures, both in the whole cohort and in nondiabetics, but not in diabetic persons.

\section{Discussion}

To our knowledge, this is the first population-based study specifically aimed at examining the association between serum bilirubin concentrations and kidney function measures in the general population. In a nationally representative sample of US adults, we found a significant, inverse association between normal serum total bilirubin concentrations and eGFR. This association was independent of a broad spectrum of established risk factors and potential confounders, such as age, gender, race/ethnicity, smoking status, hypertension, diabetes, BMI, waist circumference, plasma lipids, glucose, insulin resistance, viral hepatitis status, alcohol consumption, and albuminuria. Notably, the inverse association between serum total bilirubin and eGFR was significant in non-diabetic individuals and after excluding participants with laboratory evidence of advanced kidney dysfunction (defined as a serum creatinine concentration $>176.8 \mu \mathrm{mol} / \mathrm{L}$ ) or possible liver disease (defined as a serum bilirubin concentration $>22.23$ $\mu \mathrm{mol} / \mathrm{L})$. Similarly, we found a positive, but much weaker, association between serum total bilirubin and abnormal albuminuria in the whole cohort and in the non-diabetic subgroup. In contrast, we did not find any association between serum bilirubin with eGFR and albuminuria in diabetic persons.

These findings partly confirm and extend our recent observations in a large hospital-based sample of 2678 adult outpatients (mean age: $55 \pm 18$ years; $43 \%$ male), 210 with diabetes (14). In that study, we found that serum total bilirubin was inversely associated with eGFR in both non-diabetic $(r=-0.17 ; p<0.0001)$ and diabetic patients $(r=-0.14 ; p<0.05)$. However, no information was available on albuminuria, comorbidities, alcohol consumption and other important potential confounders (14).

The lack of a significant association between serum bilirubin and eGFR or albuminuria observed in diabetic individuals of our cohort is in contrast with the results by Fukui et al. (13). These authors found that serum total bilirubin was positively associated with eGFR, and negatively with albuminuria in a hospitalbased sample of 633 Japanese type 2 diabetic patients (mean age: $64.4 \pm 11.5$ years; $52 \%$ male). In that study, the inverse association between serum bilirubin and albuminuria (standardized $\beta$-coefficient $=-0.19 ; p<0.0001$ ) was independent of age, gender, duration of diabetes, BMI, smoking status, blood pressure, plasma lipids and glycosylated hemoglobin. However, in that study, no adjustment was made for important confounders, such as eGFR, alcohol consumption and viral hepatitis status (13).

We believe that the apparently discrepant results regarding an association, if any, between serum bilirubin and kidney function measures in the diabetic population $(13,14)$ can be largely explained by the larger sample size of the present study and, principally, by differences in the study population (for example, different distribution of age, gender, ethnicity and comorbidities) and study methodology (general population vs. hospital-based cohort).

Clearly, we must be cautious in making any causal inference, given the cross-sectional design of our study. The exact mechanism(s) linking higher serum bilirubin and decreased kidney function measures are not fully understood. The most obvious explanation for our findings is that the greater prevalence of lower eGFR in persons with higher serum bilirubin concentrations simply reflects the coexistence of established risk factors for kidney damage. However, since in our study serum bilirubin was associated with decreasing eGFR and rising albuminuria independently of several established risk factors, it is conceivable that serum bilirubin might confer an excess risk over and above the risk expected as a result of the established and more traditional risk factors. In clinical practice, an increased serum bilirubin concentration is conventionally interpreted as a marker of liver dysfunction, 
including cholestasis, drug-induced hepatitis, viral hepatitis, Gilbert's syndrome and others (22). In our study, however, serum bilirubin was significantly associated with decreasing eGFR after excluding subjects with a serum bilirubin concentration $>22.23 \mu \mathrm{mol} / \mathrm{L}$, and even after adjusting for alcohol intake and viral hepatitis status. Thus, alcohol consumption, viral hepatitis and other advanced liver diseases are unlikely to fully explain the association of serum bilirubin concentrations with worsening kidney function. In addition, as reported previously, the exclusion of persons with a serum bilirubin concentration $>22.23 \mu \mathrm{mol} / \mathrm{L}$ minimizes any significant laboratory interference by bilirubin with the measurement of serum creatinine.

It could be hypothesized that the bilirubin-kidney dysfunction association primarily reflects the association of serum bilirubin concentrations with nonalcoholic fatty liver disease (NAFLD). NAFLD is now regarded as the hepatic manifestation of the metabolic syndrome and represents the most common cause of mild to moderate increases in serum bilirubin and other liver enzymes in Western countries (23-25). NAFLD can promote atherogenic dyslipidemia and contribute to CKD pathogenesis - as well as to accelerated atherogenesis - through the release of some pathogenetic mediators from the steatotic liver, including increased C-reactive protein and other inflammatory cytokines (26). Importantly, several studies have shown that these potential mediators of vascular and kidney damage are markedly higher in patients with NAFLD than in those without (26-31), and are thought to be pathogenic factors for the development of CKD (32-35). Consistent with the hypothesis that liver inflammation (or other liverderived factors) in NAFLD may play a role in kidney disease progression, it has been shown that type 2 diabetic subjects with chronic hepatitis B virus infection were more likely to develop end-stage renal disease compared with those not infected with hepatitis $B$ virus (36). More importantly, recent studies found that NAFLD is independently associated with an increased incidence of CKD in both non-diabetic and diabetic populations $(37,38)$.

Limitations of our study include its cross-sectional design, which allows us to identify associations and should not yield any conclusions about causation. Second, NHANES only measured total and not fractionated bilirubin concentrations (thus, excluding the possibility to discriminate the Gilbert's disease from other liver disease), and performed only a single blood draw for measuring serum bilirubin, perhaps limiting the precision of the bilirubin measurement. Third, liver ultrasonography for diagnosing NAFLD was not performed. Finally, we used the eGFR instead of a directly measured GFR to assess kidney function. It is known that current GFR estimates have greater inaccuracy in populations without known CKD than in those with kidney disease. Nonetheless, current GFR estimates facilitate the detection, evaluation, and management of kidney disease. Also, many organizations recommend the use of prediction equations for the evaluation of kidney function in large epidemiologic studies and in clinical practice (18).

Despite these limitations, our analysis has several important strengths. First, it is the most comprehensive national survey to examine the associations of serum total bilirubin with kidney function and albuminuria in US adults. Second, NHANES used uniform methods to collect data on demographics, comorbidities and laboratory measures. Third, the extensive and complete data on important factors associated with kidney disease and serum bilirubin increases allows us to give an unbiased estimate for the relationship between serum bilirubin and CKD. Finally, with the design of NHANES, we are able to generalize the results to the entire US civilian non-institutionalized population.

In conclusion, our findings suggest that increasing serum total bilirubin concentrations are independently associated with decreasing eGFR and increasing albuminuria in the US adult population. Future prospective studies are required to determine the temporal nature of this association.

\section{Conflict of interest statement}

We declare that we have not accepted any funding or support from an organization that may in any way gain or lose financially from the results of your study, that we have not been employed by an organization that may in any way gain or lose financially from the results of our study, and that we do not have any other conflicting interests.

\section{References}

1. Coresh J, Selvin E, Stevens LA, Manzi J, Kusek JW, Eggers $P$, et al. Prevalence of chronic kidney disease in the United States. J Am Med Assoc 2007;298:2038-47.

2. Hsu CY, Go AS, McCulloch CE, Darbinian J, Iribarren C. Exploring secular trends in the likelihood of receiving treatment for end-stage renal disease. Clin J Am Soc Nephrol 2007;2:81-8.

3. Muntner P, He J, Hamm L, Loria C, Whelton PK. Renal insufficiency and subsequent death resulting from cardiovascular disease in the United States. J Am Soc Nephrol 2002;13:745-53.

4. Go AS, Chertow GM, Fan D, McCulloch CE, Hsu CY. Chronic kidney disease and the risks of death, cardiovascular events, and hospitalization. N Engl J Med 2004; 351:1296-305.

5. van Domburg RT, Hoeks SE, Welten GM, Chonchol M, Elhendy A, Poldermans D. Renal insufficiency and mortality in patients with known or suspected coronary artery disease. J Am Soc Nephrol 2008;19:158-63.

6. Rifkin DE, Shlipak MG, Katz R, Fried LF, Siscovick D, Chonchol $\mathrm{M}$, et al. Rapid kidney function decline and mortality risk in older adults. Arch Intern Med 2008;168:22128.

7. Schwertner HA, Jackson WG, Tolan G. Association of low serum concentration of bilirubin with increased risk of coronary artery disease. Clin Chem 1994;40:18-23.

8. Vítek L, Jirsa M, Brodanová M, Kalab M, Marecek Z, Danzig $V$, et al. Gilbert syndrome and ischemic heart disease: a protective effect of elevated bilirubin levels. Atherosclerosis 2002;160:449-56. 
9. Djoussé L, Levy D, Cupples LA, Evans JC, D’Agostino RB, Ellison RC. Total serum bilirubin and risk of cardiovascular disease in the Framingham offspring study. Am J Cardiol 2001;87:1196-200.

10. Perlstein TS, Pande RL, Beckman JA, Creager MA. Serum total bilirubin level and prevalent lower-extremity peripheral arterial disease: National Health and Nutrition Examination Survey (NHANES) 1999 to 2004. Arterioscler Thromb Vasc Biol 2008;28:166-72.

11. Breimer LH, Wannamethee G, Ebrahim S, Shaper AG. Serum bilirubin and risk of ischemic heart disease in middle-aged British men. Clin Chem 1995;41:1504-8.

12. Rosenthal P, Pincus M, Fink D. Sex- and age-related differences in bilirubin concentrations in serum. Clin Chem 1984;30:1380-2.

13. Fukui M, Tanaka M, Shiraishi E, Harusato I, Hosoda $H$, Asano $M$, et al. Relationship between serum bilirubin and albuminuria in patients with type 2 diabetes. Kidney Int 2008;74:1197-201.

14. Targher G, Zoppini G, Guidi GC, Lippi G. Relationship between serum bilirubin and kidney function in nondiabetic and diabetic individuals. Kidney Int 2009;75:863.

15. US Department of Health and Human Services, Centers for Disease Control and Prevention. National Health and Nutrition Examination Survey. http://www.cdc.gov/nchs/ nhanes.htm.

16. National Center for Health Statistics. National Health and Nutrition Examination Survey (NHANES) Analytic Guidelines. http://www.cdc.gov/nchs/about/major/nhanes/ nhanes2003_2004/analytical_guidelines.htm.

17. National Center for Health Statistics, Centers for Disease Control. Survey operations manuals, brochures, and consent documents: 1999-current NHANES. http://www. cdc.gov/nchs/about/major/nhanes/currentnhanes.htm.

18. Stevens LA, Coresh J, Greene T, Levey AS. Assessing kidney function-measured and estimated glomerular filtration rate. N Engl J Med 2006;354:2473-83.

19. National Health and Nutrition Examination Survey 2005-2006. Documentation, codebook, and frequencies, 2008. Available at http://www.cdc.gov/nchs/data/nhanes/ nhanes_05_06/biopro_d.pdf.

20. Selvin E, Manzi J, Stevens LA, Van Lente F, Lacher DA, Levey AS, et al. Calibration of serum creatinine in the National Health and Nutrition Examination Surveys (NHANES) 1988-1994, 1999, 2004. Am J Kidney Dis 2007; 50:918-26.

21. Bonora E, Targher G, Alberiche M, Bonadonna RC, Saggiani $\mathrm{F}$, Zenere MB, et al. Homeostasis model assessment closely mirrors the glucose clamp technique in the assessment of insulin sensitivity. Studies in subjects with various degrees of glucose tolerance and insulin sensitivity. Diabetes Care 2000;23:57-63.

22. Berk PD, Jones EA, Howe RB, Berlin NI. Disorders of bilirubin metabolism. In: Bondy IK, Rosenberg LE, editors. Metabolic control and disease, 8th ed. Philadelphia: Saunders, 1980.

23. Angulo P. Nonalcoholic fatty liver disease. N Engl J Med 2002;346:1221-31.
24. Clark JM, Brancati FL, Diehl AM. The prevalence and etiology of elevated aminotransferase levels in the United States. Am J Gastroenterol 2003;98:960-7.

25. de Alwis NM, Day CP. Non-alcoholic fatty liver disease: the mist gradually clears. J Hepatol 2008;48(Suppl 1): S104-12.

26. Targher G, Marra F, Marchesini G. Increased risk of cardiovascular disease in non-alcoholic fatty liver disease: causal effect or epiphenomenon? Diabetologia 2008;51: 1947-53.

27. Abiru S, Migita K, Maeda Y, Daikoku M, Ito M, Ohata K, et al. Serum cytokine and soluble cytokine receptor levels in patients with non-alcoholic steatohepatitis. Liver Int 2006;26:39-45.

28. Hui JM, Hodge A, Farrell GC, Kench JG, Kriketos A, George J. Beyond insulin resistance in NASH: TNF-alpha or adiponectin? Hepatology 2004;40:46-54.

29. Targher G, Bertolini L, Rodella S, Lippi G, Franchini M Zoppini G, et al. NASH predicts plasma inflammatory biomarkers independently of visceral fat in men. Obesity (Silver Spring) 2008;16:1394-9.

30. Chalasani N, Deeg MA, Crabb DW. Systemic levels of lipid peroxidation and its metabolic and dietary correlates in patients with non-alcoholic steatohepatitis. Am J Gastroenterol 2004;99:1497-502.

31. Haukeland JW, Damås JK, Konopski Z, Løberg EM, Haaland T, Goverud I, et al. Systemic inflammation in nonalcoholic fatty liver disease is characterized by elevated levels of CCL2. J Hepatol 2006;44:1167-74.

32. Modlinger PS, Wilcox CS, Aslam S. Nitric oxide, oxidative stress, and progression of chronic renal failure. Semin Nephrol 2004;24:354-65.

33. Kaysen GA, Eiserich JP. The role of oxidative stressaltered lipoprotein structure and function and microinflammation on cardiovascular risk in patients with minor renal dysfunction. J Am Soc Nephrol 2004;15:538-48.

34. Stenvinkel $P$, Ketteler $M$, Johnson $R J$, Lindholm $B$, Pecoits-Filho R, Riella M, et al. IL-10, IL-6, and TNF-alpha: central factors in the altered cytokine network of uremia - the good, the bad, and the ugly. Kidney Int 2005;6: 1216-33.

35. Baylis C. Arginine, arginine analogs and nitric oxide production in chronic kidney disease. Nat Clin Pract Nephrol 2006;2:209-20.

36. Cheng AY, Kong AP, Wong VW, So WY, Chan HL, Ho $C S$, et al. Chronic hepatitis $B$ viral infection independently predicts renal outcome in type 2 diabetic patients. Diabetologia 2006;49:1777-84.

37. Chang Y, Ryu S, Sung E, Woo HY, Oh E, Cha K, et al. Nonalcoholic fatty liver disease predicts chronic kidney disease in non-hypertensive and non-diabetic Korean men. Metabolism 2008;57:569-76.

38. Targher G, Chonchol M, Bertolini L, Rodella S, Zenari L, Lippi G, et al. Increased risk of CKD among type 2 diabetics with nonalcoholic fatty liver disease. J Am Soc Nephrol 2008;19:1564-70. 